\title{
Définir le mercenaire puis lutter contre le mercenariat
}

\section{Christian Leverchy}

\section{OpenEdition}

\section{Journals}

Édition électronique

URL : http://journals.openedition.org/conflits/979

DOI : $10.4000 /$ conflits. 979

ISSN : $1777-5345$

Éditeur :

CCLS - Centre d'études sur les conflits lilberté et sécurité, L'Harmattan

Édition imprimée

Date de publication : 1 décembre 2003

Pagination : 67-90

ISBN : 2-7475-6065-1

ISSN : 1157-996X

Référence électronique

Christian Leverchy, « Définir le mercenaire puis lutter contre le mercenariat », Cultures \& Conflits [En ligne], 52 I hiver 2003, mis en ligne le 03 juillet 2004, consulté le 30 mars 2021. URL : http:// journals.openedition.org/conflits/979; DOI : https://doi.org/10.4000/conflits.979

Ce document a été généré automatiquement le 30 mars 2021.

Creative Commons License 


\title{
Définir le mercenaire puis lutter contre le mercenariat
}

\author{
Christian Leverchy
}

De gauche à droite sur les bancs de l'Assemblée nationale et du Sénat ou à longueur de colonnes de presse s'exprime, dorénavant, le consensus selon lequel l'utilisation de mercenaires dans les conflits armés ou les situations troublées est un phénomène qui aggrave la violence. ${ }^{1}$ Les nouvelles générations de lansquenets déstabiliseraient non seulement les Etats mais génèreraient même mécaniquement des atteintes aux droits de l'homme par le recours régulier à des trafics illicites d'armement notamment d'armes légères et de petit calibre (ALPC). Dès lors, légiférer contre cette pratique serait devenu urgent, indispensable mais aussi possible. Il n'en demeure pas moins que cette présente posture politique et morale ne peut faire oublier l'attitude ambivalente que nombre d'Etats ont adopté au fil du temps vis-à-vis de ces soldats de fortune.

La condamnation morale actuelle n'est pas pour autant totalement nouvelle. Signe des temps alors que la première édition du dictionnaire de l'Académie française (16942) insistait surtout sur le caractère vénal des "Affreux ", les parlementaires français reprenant les slogans des organisations citoyennes qui militent depuis quelques années pour la ratification de la convention internationale contre le recrutement, l'utilisation, le financement et l'instruction des mercenaires du 4 décembre 1989 - se déclarent, aujourd'hui, convaincus qu'un conflit armé serait plus criminogène si d'aventure l'une des parties décidait d'admettre à son service ces combattants privés. Les comportements pénalement répréhensibles (tels que : crimes de guerre, blanchiment de l'argent sale, corruption, exploitation indue des ressources naturelles rares...) de ces soldats d'appoint seraient donc inhérents à leur métier alors que seulement certains d'entre eux seront justiciables devant la nouvelle Cour pénale internationale.

Rien n'est moins sûr que ce caractère criminogène des mercenaires contemporains, agissant individuellement ou collectivement ! Cependant, la conviction est si largement partagée que plusieurs parlementaires ${ }^{3}$ avaient souhaité inclure dans la loi française réprimant les activités des mercenaires une punition «exemplaire» de leurs commanditaires politiques locaux. En attendant, l'adoption à l'unanimitét ${ }^{4}$ et après des 
retouches «mineures » du projet de loi présenté au nom du gouvernement de L. Jospin par le ministre de la Défense, Alain Richard, au Sénat, le 3 avril 2002, témoigne qu'il existe de part et d'autre du spectre politique national une même volonté d'afficher sa détermination contre l'usage des mercenaires. Reste à savoir si celle-ci conduit à élaborer des politiques publiques mimétiques! Les uns ne voient-ils pas dans ce texte législatif un document pour solde de tout compte? La ministre de la Défense a ainsi déclaré au cours des débats à l'Assemblée nationale qu'il "convient d'encadrer le phénomène du mercenariat en sanctionnant ses manifestations les plus condamnables, mais sans entraver toute possibilité de renforcer la protection de l'Etat $»^{5}$. Les autres n'exprimentils pas le sentiment diffus que les mesures adoptées sont déjà en retard sur l'évolution des réalités qu'elles désignent ? Perplexes quant à l'efficacité du dispositif retenu, ils se demandent vraiment pourquoi avoir exclu des contours répressifs de la loi les activités de formation, de préparation opérationnelle et d'entraînement conduites par les mercenaires. En bref, ces observateurs dubitatifs s'interrogent sur la détermination réelle de l'Etat français à réprimer le phénomène. Une inquiétude nourrie des expériences passées. Les mercenaires n'ont-ils pas constamment bénéficié d'une impunité quasi-totale de la part des gouvernements successifs de leur pays d'origine comme le soulignent à intervalles réguliers tout particulièrement la presse et les dirigeants africains ${ }^{6}$ ? Dans un contexte mémorial si chargé et une prise de conscience si tardive de la nécessité de combattre les activités des mercenaires, cette lutte ne peut souffrir dorénavant le moindre atermoiement.

Une prise de conscience tardive

Désormais, l'emploi de l'épithète de "mercenaire" donne matière à la vindicte populaire. Comme l'a souligné J. A. Schumpeter, l'esprit commerçant de nos sociétés pacifiées a contribué à la genèse et à la propagation d'une mentalité post-héroïque qui est devenue prédominante, dévalorisant la fonction utilitaire des mercenaires d'autrefois. De facto, celle-ci a écarté puis marginalisé les groupes belliqueux et violents. C'est pourquoi, au cours des discussions lors de l'adoption de la nouvelle loi, parlementaires de l'opposition et de la majorité ont donc fait chorus pour dénoncer le phénomène et souligner les carences manifestes du droit pénal français et l'absence de moyens légaux pour combattre efficacement ces pratiques d'un autre âge même s'il existe un droit international coutumier qui condamne et interdit ce type d'activités, du seul fait de leur nature.

Alors que les débats interministériels lors de l'écriture du projet de loi ont été intenses entre les administrations voire conflictuels ${ }^{7}$, la définition large retenue n'a étonnement pas fait l'objet de longues délibérations parlementaires ou de commentaires critiques. Le dispositif qui concerne aussi bien les conflits inter étatiques qu'intra étatiques n'a pas généré plus de polémiques. Plus surprenant, personne ne s'est étonné de ce que le texte supprime les conditions habituellement exigées pour poursuivre des Français ayant commis des délits hors du territoire national et étende, pour la seconde fois, la compétence des tribunaux français selon la jurisprudence des crimes sexuels.

Cette absence de controverses récentes s'explique notamment par le sentiment général d'une urgence impérieuse, révélée par les derniers soubresauts des crises africaines et la certitude d'être devant un phénomène en recrudescence. En outre, il se dégage comme une évidence que cette fourniture de prestations militaires contribue à la marchandisation des conflits. S'y montrer bienveillant constituerait non seulement une faute morale mais un danger militaire pour les forces françaises. L'impossibilité de 
poursuivre et condamner à une peine adéquate les aventuriers français qui ont tenté de s'emparer de l'île de Mohéli (Comores) en décembre 2001 autrement qu'en les incriminant pour association de malfaiteurs fut un révélateur tout aussi efficace que le recours croissant de l'armée régulière ivoirienne à ces "spécialistes" au cours des dernières années. Les difficultés de sanctionner les activités désordonnées du général (c.r.) Jeannou Lacaze suscitèrent le même malaise. Autant de raisons qui expliquent que c'est le ministère de la Défense qui appela de ses vœux une nouvelle législation et son Ministre qui défendit le projet de loi en Conseil des ministres puis devant les Assemblées.

Avec solennité et gravité, il est apparu aux élus qu'il convenait, non seulement de prévenir mais aussi de réprimer toute implication de ressortissants français dans ce type d'activités. L'enjeu serait même devenu si important qu'il est susceptible de mettre en cause la crédibilité de l'Etat français. Faudra-t-il par conséquent le mesurer précisément à l'aune du nombre de procédures engagées ${ }^{8}$ ? En attendant, effet de dramatisation oblige, pour reprendre les termes de la ministre de la Défense: «le mercenariat est un phénomène dangereux, qui contribue à pérenniser des situations de crise dans les Etats les plus fragiles " ${ }^{9}$. Ce constat est pour le moins contradictoire avec les réalités observées mais il n'en est pas moins répété, urbi et orbi, comme si l'on recherchait obstinément tous les arguments nécessaires pour justifier l'adoption de la loi et se convaincre que celle-ci mettra à elle seule fin à l'épiphénomène.

L'emploi de ces « marchands de chaos » n'a de sens que s'ils apportent rapidement une solution militaire voire définitive aux difficultés rencontrées par leurs employeurs. Pour s'opposer à toutes les activités militaires supervisées par des mercenaires, la puissance publique se doit de disposer de plusieurs instruments de coercition. En outre, en situation de crise aiguë, elle dispose de peu de temps pour réagir. Elle doit par conséquent inventer des procédures juridiques et administratives immédiatement contraignantes pour faire obstacle aux déploiements opérationnels des mercenaires. $\mathrm{Si}$ ceux-ci contreviennent directement aux intérêts de l'Etat, il s'agit de faire perdre du temps à ceux qui offrent leurs services, bref de leur compliquer la vie. La loi votée en 2003 n'a donc de sens que prise dans un ensemble de mesures susceptibles de limiter l'accès des mercenaires aux moyens militaires dont ils ont besoin (par exemple, des contrôles renforcés des exportations d'armement à destination des acteurs non gouvernementaux et des courtiers).

D'expériences, le temps apparait comme une valeur essentielle de l'action des mercenaires. Or, s'il est encore rare d'observer que des mercenaires s'opposent les armes à la main à leurs compatriotes ou s'enrôlent comme autrefois tôt ou tard dans les rangs de leurs adversaires, la fin de la guerre froide, la réduction du format des armées voire leur professionnalisation croissante ont augmenté de manière significative le volume de main d'œuvre disponible. Non seulement le stock de soldats intérimaires, «jeunes» et rapidement disponibles appert croissant mais les mercenaires " charismatiques » à la recherche de collaborateurs compétents bénéficient dorénavant de nouvelles structures entrepreneuriales performantes, nées de la privatisation des forces armées ${ }^{10}$. Cette réalité préoccupe d'autant plus les états-majors que les mercenaires sont particulièrement rompus aux actions de guérilla et mettent en danger plus que jamais la vie de leurs personnels ${ }^{11}$. Les mercenaires accroissent les capacités belligènes des forces qu'ils encadrent. Ils introduisent non seulement leur savoir-faire militaire généraliste (instruction, encadrement, entre autres) ou spécialisé 
(déminage ${ }^{12}$, transmission, renseignement, artillerie, opérations spéciales...) mais exigent également des matériels plus performants à leur disposition sur les théâtres d'opération. Ainsi, Ukrainiens et Russes se sont fait une spécialité de services héliportés, matériels particulièrement bien adaptés aux stratégies de contreinsurrection. Ils ne sont pas pour autant une garantie absolue de succès comme en ont témoigné les déconvenues du gouvernement zaïrois, en 1996, avec sa «Légion blanche ».

Les mercenaires peuvent porter atteinte à la sécurité des forces françaises, de ses personnels, bref à la mission que l'Etat assigne à ses hommes. Plus grave, il devient difficile de prendre des distances par rapport à leurs actions faute de pouvoir émettre un démenti plausible. Alors que les mercenaires qui se présentent volontiers comme les "Corsaires de la République " sont accusés d'entretenir des relations consanguines avec leur armée nationale, cette confusion est dorénavant jugée préjudiciable aux intérêts de la France, surtout lorsqu'elle intervient dans des conflits où ses propres armées se trouvent impliquées, directement ou par l'entremise des actions interétatiques de coopération et/ou des services de renseignement. Ainsi, C. Josselin et A. Richard conditionnèrent la reprise de la coopération de défense avec le CongoBrazzaville à la cessation des activités des mercenaires recrutés par le président $\mathrm{D}$. Sassou Nguesso. Tout aussi préjudiciable est le soupçon nourri sur l'attitude des services de renseignement vis-à-vis des mercenaires. S'ils peuvent servir d'échotiers aux argousins comme aux services spéciaux et aux commerciaux des sociétés d'armement ${ }^{13}$, il convient de veiller qu'une attitude passive ne se transforme en une instrumentalisation mutuelle. Pour éviter des relations de trop grandes proximités avec les services de renseignement extérieurs ${ }^{14}$ et intérieurs (par exemple, DPSD) encore faut-il prendre conscience des risques politiques encourus. Ceux-ci qui pèsent autant sur les personnels engagés en conditionnant négativement leur espace d'intervention que sur l'efficacité de la diplomatie française. L'accentuation du contrôle des services de renseignement ne doit pas plus se traduire tôt ou tard par le recours de ceux-ci à des sociétés privées afin d'éloigner les investigations sur les activités les plus sensibles conduites à l'étranger. Afin de remédier à une telle dérive, il importe que les autorités politiques assument mieux pour ne pas dire pleinement toutes les activités des services de renseignement sur lesquels ils exercent une autorité. Privatiser le recours à la force pour ne pas assumer ses responsabilités comme on a pu le constater aux Etats-Unis ${ }^{15}$ est des plus problématiques.

Faire obstacle aux mercenaires, c'est également contribuer à l'adoption par les partenaires de coopération des symboles refondateurs de la bonne gouvernance. En Côte d'Ivoire, ordre fut ainsi donné d'abattre les hélicoptères engagés par les mercenaires recrutés par le Président L. Gbagbo. Pour l'Etat français, ces gestes visent à ne pas être incriminés plus que de raison. Plus que jamais l'Etat est soucieux de son image notamment là où l'on lui prête une influence politique prépondérante. L'Afrique apparaissant comme le continent de prédilection des activités de mercenariat ${ }^{16}$, la lutte contre ces pratiques est apparue au gouvernement de L. Jospin comme l'un des symboles de la redéfinition urgente de la politique de coopération. Il s'agissait ni plus, ni moins que de tourner définitivement les pages les plus sombres du passé, mises en exergue à la même période par la mission parlementaire sur le Rwanda et de faire obstacle à une praxis géographiquement de plus en plus diversifiée (Casamance, 1996; Congo-Brazaville, 1997; République démocratique du Congo, 1996; Comores, 1975, 1978, 2001; Côte d'Ivoire, 2000, 2002) voire plus lointaine encore (Birmanie, 1995'77). 
Afghanistan oblige, se doter de nouveaux moyens juridiques pour se prémunir ex-ante des actions des mercenaires pouvait s'avérer également utile dans la lutte d'ampleur nouvelle qui s'engageait au même moment contre les groupes terroristes transnationaux.

Dans ce contexte, la lutte contre le mercenariat est devenue un projet de gouvernement. Sur le plan éthique, il s'agissait d'afficher ainsi la nécessité pour la France de contribuer à la construction d'un ordre international respectueux des droits de l'homme. A cette fin, dans sa déclaration de politique générale, le 19 juin 1997, le Premier ministre L. Jospin a déclaré ${ }^{18}$ que : «[le] gouvernement procèdera à un réexamen attentif de tous les instruments internationaux relatifs aux droits de l'homme auxquels la France n'est pas partie en vue d'y adhérer ». Cette politique s'inscrivait dans une vision d'un socialisme réformiste, ni anti-militariste, ni pacifiste mais qui soulignait que le recours à la force devait s'inscrire dans des règles de droit précises. Preuve de cet attachement aux règles patiemment esquissées, le groupe socialiste de l'Assemblée nationale déposa le 18 décembre 2002, à l'instigation de MM. Paul Quilès et Jean-Marc Ayrault, une proposition de loi $\left(\mathrm{n}^{\circ} 499^{19}\right)$ reprenant à l'identique le projet promu par le précédent gouvernement.

Contrairement à une idée reçue, ce texte n'a pas été examiné d'abord au Sénat pour des raisons de calendrier. Le choix de la Haute assemblée fut essentiellement politique, pour ne pas dire tactique. Faute d'avoir pu respecter le calendrier de travail interministériel fixé, le projet de loi initial de L. Jospin adopté en Conseil des ministres visait à mettre la nouvelle majorité parlementaire devant ses responsabilités. Agréé par le chef de l'Etat en conseil des ministres, elle se devait d'engager son éventuelle majorité. En déposant le texte sur le bureau du Sénat, son cheminement législatif ne pouvait être interrompu par les échéances électorales de l'année 2002. L'abandonner ne pouvait se faire sans payer un coût politique.

Conçu comme un outil irénologique, le projet de loi s'est voulu d'emblée ambitieux. Une ambition qui a été explicitement affichée dans le titre des trois chapitres du vecteur législatif. Là une définition claire se justifiait comme un continuum à la ratification le 11 avril $2001^{20}$ du protocole I du 8 juin 1977 additionnel aux conventions de Genève du 12 août 1949. Puisque la France reconnaissait comme répréhensible l'activité du mercenaire, elle se devait de sanctionner précisément cette activité. L'article 436-1 du code pénal introduit par le projet de loi «mercenariat » qui définit l'activité répréhensible et caractérise la peine, n'a donc fait que reprendre les dispositions du protocole I. Les sanctions pénales importantes ont été établies au maximum du possible. Par souci de cohérence aussi, elles ont été fixées à hauteur de celles décidées pour la violation de la convention chimique et lors de la transcription en droit interne de la convention d'Ottawa sur les mines antipersonnel terrestre. Quant au large champ d'application (et contre l'avis de beaucoup), le projet d'article 436-3 a prévu l'extension de la compétence des juridictions pénales françaises puisqu'il vise à réprimer les actes commis par des Français à l'étranger et des personnes résidant habituellement sur le territoire français. Un choix né des expériences les plus récentes ou nombre d'opposants africains résidents en France (Comores, Côte d'Ivoire, RCA, RDC...) ont organisé depuis le territoire national et le plus souvent dans les grands hôtels de la capitale le recrutement de soldats de fortune d'ailleurs pas nécessairement français (Serbes, Roumains,...) pour renverser leur gouvernement. 
L'adoption d'une loi spécifique ne s'est donc pas faite immédiatement. D'ailleurs les organisations de vigilance citoyenne (entre autres, Survie ${ }^{21}$ ) et plusieurs députés de la majorité tel P. Brana (PS), A. Gérin (PCF) ou le président de la commission des Affaires étrangères J. Lang (6 septembre 1999) appelèrent d'abord de leurs vœux la ratification de la convention internationale contre le recrutement, l'utilisation, le financement et l'instruction des mercenaires du 4 décembre 1989. Cette ratification se posait avec d'autant plus d'acuité que la convention est entrée en vigueur pendant la législature (20 octobre 2001) et qu'elle fut ratifiée, de 1997 à 2002 par onze de ses vingt-quatre signataires. On peut donc dire que le choix d'une loi spécifique aux mercenaires est né tant des difficultés particulières soulevées par la convention de 1989 que de la dynamique réformatrice du gouvernement - déterminé à transposer rapidement en droit interne les instruments internationaux relatifs aux droits de l'homme récemment adoptés et animé d'une farouche volonté de lutter efficacement contre les trafics d'armement (et, en premier lieu, ceux constitués de cargaisons d'ALPC). Ceteris paribus, l'adoption par l'Afrique du Sud (Regulation Foreign Military Assistance Act, 26 février 1998) d'un dispositif législatif original et les réflexions britanniques ${ }^{22}$ sur l'encadrement réglementaire des sociétés privées de sécurité ne furent pas non plus étrangères à ce processus de maturation.

Pour autant, le dépôt du projet de loi n'est pas exempt de calcul partisan, voire d'a priori idéologiques. A gauche, on reste convaincu que ce sont des mercenaires français impliqués dans l'assassinat du président comorien Ahmed Abdallah (novembre 1989) qui ont également assassiné à Paris la représentante de l'A.N.C., Dulcie September, le 29 mars 1988. Par conséquent, ce texte visait également d'une certaine manière à lutter contre certains réseaux d'extrême-droite, soupçonnés de bénéficier d'une bienveillante neutralité auprès de certains services du ministère de la Défense. En décembre 1997, le réseau « Voltaire » avait adressé en ce sens un dossier aux députés et leur demandait de constituer une commission d'enquête parlementaire sur le « service d'ordre » du Front National, le DPS(Département Protection Sécurité23) mettant en cause notamment l'exdirecteur des services techniques de la ville de Vitrolles, François-Xavier Sidos (FN), bien connu pour ses activités aux Comores auprès de Bob Denard. S'il fallait déceler une preuve de cette collusion entre le monde mercenaire et celui de l'extrême-droite, un ancien responsable du Groupe Union Défense(GUD), Richard Rouget, en a donné un exemple bien malgré lui. En 2003, il fut la première personne condamnée par la justice sud-africaine en vertu de la loi de 1998 interdisant toute activité mercenaire d'assistance à un conflit étranger par les Sud-Africains ou sur le sol sud-africain.

Les conditions politiques étant réunies pour une initiative législative encore fallait-il faire attention aux malentendus sémantiques. Que voulait-on réprimer? Il ne s'agissait bien évidemment pas de viser tous les fonctionnaires de l'Etat pour reprendre la définition pour le moins extensive du mercenariat donnée au début du siècle dernier par Rosa Luxembourg (L'accumulation du capital,1913) à la suite de Karl Marx qui qualifiait de son côté de mercenariat les formes embryonnaires du travail salarié. Audelà de la criminalisation des mercenaires pour leur statut, il fut décidé de considérer ni plus ni moins ces hommes comme des armes conventionnelles que l'on se devait de contrôler en tant que telles. C'est si vrai que le premier document public précisant les intentions du gouvernement et son calendrier de travail fut le deuxième rapport annuel du ministère de la Défense sur les exportations d'armement ${ }^{24}$. Autrement dit, le contrôle des mercenaires s'est ainsi inscrit dans le cadre plus général de l'amélioration $\mathrm{du}$ dispositif administratif, réglementaire et législatif du contrôle des exportations 
d'armement voulu par le ministre de la Défense. Respectant la même démarche réformatrice, préférant améliorer les textes existants à l'innovation législative permanente, et le calendrier d'ensemble.

Les règles existantes de droit français et de droit international étaient inappropriées

La définition du cadre juridictionnel visant à combattre le mercenariat est née, en 1997, des échanges interministériels sur la possibilité pour la France de ratifier le protocole I du 8 juin 1977 additionnel aux conventions de Genève du 12 août 1949. Ces échanges ont convaincu le ministère de la Défense et le cabinet du Premier ministre que ce vecteur n'était pas le plus approprié. En effet, il «n'est en aucun cas un instrument de lutte contre la pratique tendant à employer comme militaire des étrangers soldés " pour reprendre les termes exprimés ultérieurement par le rapport du député Marc Jourlaud ${ }^{25}$. Ce constat $n$ 'en mit pas moins en évidence que jusqu'ici la notion de mercenariat, dont les caractéristiques paraissaient difficiles à cerner par ailleurs ${ }^{26}$, n'existait pas en tant que telle en droit français. Il en allait de même dans le droit international, chez la plupart des Etats étrangers et nos principaux alliés.

En France, les codes civil et pénal comportaient trois dispositions particulières, jugées après réexamen unanimement inappropriées. D'un côté, l'article 23-8 du code civil stipule que «perd la nationalité française le Français qui, occupant un emploi dans une armée ou un service public étranger ou dans une organisation internationale dont la France ne fait pas partie ou plus généralement leur apportant son concours, n'a pas résigné son emploi ou cessé son concours nonobstant l'injonction qui lui en aura été faite par le Gouvernement ». De l'autre, l'article 25 du code civil précise que peut être déchu de la nationalité française tout individu qui s'est livré à des actes incompatibles avec la qualité de Français et préjudiciables aux intérêts de la France. Quant à l'article 413-1 du code pénal, il réprime, lui, le fait d'inciter des militaires français à passer au service d'une puissance étrangère. Même si les peines prévues peuvent aller jusqu'à dix ans d'emprisonnement et un million de francs d'amende, ce cas d'école ainsi réprimé comme les deux précédents ne s'applique que de manière rarissime aux mercenaires.

Par ailleurs, le droit international n'était pas plus précis et d'un meilleur recours. Les activités de mercenariat ne sont incriminées ni par la cinquième convention de La Haye de 1907 (article 17), ni dans la troisième convention de Genève du 12 août 1949 (article 4). Ces dispositions ont même pour conséquence le fait qu'un mercenaire devrait pouvoir bénéficier du traitement réservé aux prisonniers de guerre. A contrario,le protocole I de 1977 précise que les mercenaires n'ont droit ni au statut de combattant, ni au statut de prisonnier de guerre. Paradoxalement, c'est donc la ratification de ce texte « excluant » qui va servir de catalyseur pour les étapes suivantes. L'article 47 et ses six critères cumulatifs ont permis de définir le mercenaire de façon stricte. Restait donc à savoir si la convention internationale contre le mercenariat adoptée le 4 décembre 1989 lors de l'Assemblée générale des Nations-Unies était plus appropriée avant d'envisager définitivement la rédaction d'une nouvelle loi. Dès le premier abord, il est apparu qu'elle définissait le mercenariat d'une façon vague et donc difficilement acceptable par la plupart des Etats. De ce fait, seul un petit nombre d'entre eux - et aucun membre permanent du Conseil de sécurité - est partie à cette convention. Ce texte pose de réels problèmes pratiques notamment du fait de l'incertitude quant à l'inclusion de la Légion étrangère dans son champ d'application ${ }^{27}$.

La ratification de la convention de 1989, une «non » solution 
Le 4 décembre 1989, l'Assemblée générale des Nations-Unies a adopté sans vote la convention contre le mercenariat, après dix années de laborieuses négociations amorcées, en 1979, à l'initiative du Nigéria. L'élaboration de ce texte fut l'occasion de vives polémiques politiques entre certains pays en voie de développement (Cuba, Angola, Nicaragua, Afghanistan...) soutenus par les pays du camp socialiste, et les pays occidentaux. Toutefois, l'examen interministériel de ce texte fut l'occasion de la maturation de la réflexion du gouvernement sur la définition précise à retenir du mercenaire. En effet, la convention, dans son article premier, en donne une définition intéressante. Sont mercenaires au sens de la convention les personnes qui prennent part aux combats dans un conflit armé si elles sont spécialement recrutées pour ce faire et si elles prennent part aux hostilités essentiellement en vue d'obtenir un avantage personnel et qu'il leur ait promis une rémunération matérielle nettement supérieure à celles des autres combattants de l'Etat qui les a recrutés. Il faut également qu'elles ne soient pas membres des forces armées de l'Etat en question et qu'elles ne soient pas envoyées en mission officielle par un autre Etat en tant que membre de ses forces armées. A contrario, dans toute autre situation que les conflits armés, le mercenaire s'entend, par conséquent, de toute personne qui est recrutée pour prendre part à une action concertée de violence conçue pour atteindre un ou plusieurs des objectifs suivants : renverser un gouvernement, ébranler l'ordre public, mettre en danger la sécurité des populations, endommager des biens publics ou des biens privés, intervenir dans les affaires intérieures d'un Etat, porter atteinte à l'intégrité territoriale et à l'indépendance d'un territoire. Son état est donc établi s'il prend une part directe à de telles actions, s'il agit essentiellement en vue d'obtenir une rémunération matérielle nettement supérieure à celle de personnes ayant un rang et des fonctions analogues dans les forces armées de l'Etat dont elle a la nationalité, s'il n'est pas ressortissant de l'Etat contre lequel ces actes sont dirigés, s'il n'est pas membre des forces armées de cet Etat et s'il n'a pas été envoyé par un Etat en mission officielle en tant que membre des forces armées dudit Etat. Autant de conditionnalités cumulatives qui ont été étudiées puis retenues pour le projet de loi français. En outre, dans ses articles 2, 3 et 4, la convention interdit donc à toute personne de recruter, financer, instruire ou employer des mercenaires ou de se rendre complice de tels actes, sous peine de commettre une infraction. Elle interdit au mercenaire de participer à un conflit armé ou à un acte concerté de violence. Cette convention fait donc obligation aux Etats de ne pas recourir aux mercenaires de façon générale, en particulier en vue de s'opposer à l'exercice légitime du droit des peuples à l'autodétermination, sous peine d'engager leur responsabilité internationale. La convention incite également les Etats à organiser une coopération entre eux en vue de prévenir et de réprimer les infractions commises.

La convention, ouverte à la ratification jusqu'au 31 décembre 1990, n'a été signée que par de très rares partenaires militaires d'importance de l'Etat français (Italie, 1995; Belgique, 2002) et un seul Etat africain francophone, le Togo. L'Italie et la Belgique, comme l'Allemagne (qui a signé la convention mais ne l'a pas ratifiée), n'ont ni territoire, ni département d'outre-mer, ni unités équivalentes à la Légion étrangère française. Ils ne déploient en outre qu'exceptionnellement des forces militaires en dehors de leur propre territoire même si la construction de l'Europe de la défense fait déjà évoluer cette réalité. Il est par contre significatif de rappeler que l'Espagne et le Royaume-Uni, qui disposent d'unités composées de ressortissants étrangers (Légion étrangère espagnole, Gurkhas britanniques) n'ont pas adhéré à la convention, ni manifesté l'intention de le faire. 
Jusqu'ici la France avait donc toujours adopté une position très réservée sur ce texte. La définition insuffisamment précise $d u$ concept de mercenaire soulevait de réelles interrogations. Il n'est pas absolument certain juridiquement que cette définition puisse interdire à un Etat étranger partie de s'en prévaloir pour considérer que les soldats de nationalité étrangère servant ainsi la France au sein de la Légion étrangère sont assimilables à des "mercenaires ", au regard de leur statut particulier. Surtout, la définition du mercenaire donnée par la convention ne permettait pas d'affirmer avec certitude que les Français présents dans des sociétés de service dépendant du ministère de la Défense, ou ceux servant au titre de l'assistance militaire technique à l'étranger pour assurer des missions d'instruction, d'organisation, d'entraînement ou d'encadrement au profit des forces armées étrangères ne pourront pas être assimilés tôt ou tard à des mercenaires, s'ils venaient à tomber entre les mains d'un Etat étranger en conflit avec celui auprès duquel ces personnes sont placées. En effet, les dites personnes ont des statuts très variés. Certaines sont des militaires en activité, détachés dans des sociétés de service ou directement auprès de l'Etat étranger, souvent d'office (pour éviter qu'ils ne perdent certains avantages liés à leurs grades et emplois). D'autres, en revanche, sont d'anciens militaires français, qui obtiennent ces emplois en raison de leurs compétences passées, par recommandation des services officiels, soit dans ces sociétés de service, soit directement auprès d'un gouvernement «ami ». Or, il est arrivé à toutes ces catégories de personnes d'être amenées à prendre part à des hostilités. Pour la deuxième catégorie mentionnée (les personnes qui ne sont plus des militaires au sens du statut de 1972), il pourrait être difficile de démontrer, à l'appui de leur défense et de celle de l'Etat français, que d'une part ils ne répondent pas à la définition du mercenaire et que d'autre part la France n'a pas manqué à ses obligations en aidant à leur recrutement. Les règles de procédure établies par la convention pourraient amener l'Etat français à devoir communiquer des informations couvertes par le secret de défense, notamment pour prouver qu'une personne appartient bien aux forces armées françaises ou se trouve réellement en mission officielle. Une construction de preuve d'autant plus difficile que la ministre de la Défense, Mme AlliotMarie, a reconnu lors du débat parlementaire que « toute personne envoyée en mission par l'Etat ne l'est pas toujours de manière officielle ou officialisée $»^{28}$. Nonobstant ce rappel plein de franchise, la substitution par les sénateurs de l'expression « mission officielle » par le mot « mission » dans le corps de la loi est donc lourde de conséquence voire de sens. En l'absence d'autorités régulatrices rien ne permet de savoir si des missions peuvent être agréées pour d'autres acteurs que l'Etat. Le président de la commission de la défense de l'Assemblée nationale, M. Guy Tessier, a lui-même regretté l'absence de "statut juridique spécifique de ces missions, confiées de gré à gré à d'anciens officiers français " même si elles s'apparentent "beaucoup plus à des missions de coopération militaire qu'à des actes de mercenariat $»^{29}$. Tout en craignant que ce vide juridique ne s'avère préjudiciable à ces personnels (le terme étant ambigu peut laisser penser qu'ils sont en service), rien n'a été proposé.

Si la France n'envisage plus du tout, aujourd'hui, de signer la convention de $1989^{30}$, le gouvernement de L. Jospin n'avait, lui, pas totalement écarté l'idée d'adhérer à cet instrument $^{31}$. Si ce choix avait été retenu définitivement, il aurait, toutefois, nécessité de formuler des déclarations interprétatives et des réserves en bonne et due forme. Celles-ci étaient jugées indispensables à la préservation tant des intérêts des membres des forces armées que des personnes engagées à l'étranger. La convention n'empêche pas la formulation de telles déclarations et réserves. L'élaboration de ces réserves se 
devait en tout état de cause se dérouler dans le cadre d'un long et délicat processus de consultation interministériel. De plus, un tel processus aurait nécessité, lui aussi, une procédure d'adhésion parlementaire, en vertu de l'article 53 de la constitution. Cette démarche, contrairement au cas des Etats qui avait déjà signé la convention avant le 31 décembre 1990, aurait dû s'effectuer en une seule étape (adhésion) et non en deux (signature puis ratification). Toute réserve ou déclaration ne pouvait être faite au plus tard qu'au moment du dépôt de l'instrument d'adhésion. La France n'aurait pu par conséquent se prévaloir d'exemple de réserves exprimées par ses devanciers. La nécessité de mettre au point celles-ci risquaient au demeurant de limiter la portée du geste politique et de susciter plus de suspicions encore sur la détermination réelle de l'Etat français à combattre ce phénomène. En outre, l'adhésion supposait très vraisemblablement l'adoption d'une loi interne, notamment pour permettre aux tribunaux français d'exercer la compétence universelle organisée par la convention, dès lors que celle-ci entrerait en vigueur. Bref, une procédure longue et politiquement bien peu avantageuse!

Il devenait ainsi de plus en plus évident qu'une loi particulière s'imposait sauf à trouver dans les obligations statutaires pesant sur les anciens militaires français un dispositif permettant de contrecarrer cette évolution très particulière de leur carrière.

Les obligations statutaires pesant sur les anciens militaires français sont inappropriées pour lutter contre le mercenariat

Les obligations statutaires pesant sur les anciens militaires français ne se sont pas avérées plus appropriées pour combattre le mercenariat. L'article 35 de la loi du 13 juillet 1972 portant statut général des militaires se contente de préciser que: "Conformément aux dispositions du code pénal, les militaires de carrière ne peuvent avoir par eux-mêmes ou par personne interposée, sous quelque forme que ce soit, tant qu'ils sont en activité ou pendant un délai de cinq ans à compter de la cessation des fonctions, dans les entreprises soumises à leur surveillance ou à leur contrôle ou avec lesquelles ils ont négocié des contrats de toute nature, des intérêts de nature à compromettre leur indépendance». Ces dispositions sont un rappel de celles de l'article 432-13 du code pénal. Elles sont applicables à tous les militaires. L'article 1 du décret du 11 janvier 1996 n'est pas d'un plus grand secours. Il se limite à préciser que certaines catégories de militaires, dont les officiers généraux admis en deuxième section doivent informer l'administration du ministère de la Défense de la nature de leur nouvelle activité privée lucrative lorsqu'ils cessent définitivement leurs fonctions ou ont cessé définitivement celles-ci depuis moins de cinq ans. Le ministre dispose alors d'un délai de deux mois pour leur signifier, après saisine de la commission de déontologie, si cette activité est compatible avec les dispositions de l'article 35 de la loi du 13 juillet 1972. On voit mal un citoyen déclarer une activité devenue illégale.

Les autres mesures réglementaires n'étaient pas plus efficaces. Indépendamment des dispositions du code pénal relatives à la violation du secret professionnel, l'ensemble des militaires, y compris les officiers généraux en deuxième section, est lié par des obligations de réserve et de discrétion (article 7 et 87 de la loi du 13 juillet 1972). Ils ne peuvent donc, après avoir quitté l'armée, divulguer des informations dont ils ont eu connaissance dans leurs fonctions militaires. Les mercenaires n'ont pas besoin de révéler les secrets des armées françaises sauf s'ils sont recrutés à cette seule fin, un scénario plausible mais peu réaliste au regard des dernières décennies. 
Les obligations statutaires se sont révélées d'autant plus inappropriées pour lutter contre le mercenariat qu'elles se sont montrées inégalitaires entre les personnels et visent tout particulièrement les officiers généraux. Force est de constater que ces derniers ne constituent pas l'essentiel du corps de troupe visé. Ainsi, les officiers généraux admis en deuxième section, mais pas les autres anciens militaires, sont liés par les dispositions de l'article 48 de la loi du 13 juillet 1972 celui-ci prévoyant que des sanctions peuvent être prononcées en cas de faute contre l'honneur.

Autre preuve d'inadaptation des textes à cette nouvelle réalité économique, la non prise en compte des nouvelles données entrepreneuriales. L'exercice par des anciens militaires français d'une activité lucrative exercée au bénéfice d'une société étrangère n'est pas interdit ni donc réprimé par le droit français, ni par les normes du droit international auxquelles la France est partie prenante. De même, les obligations statutaires n'interdisent pas de telles activités. Il n'en irait autrement si le statut général de 1972 était modifié par exemple pour interdire l'exercice d'une activité auprès d'un gouvernement ou d'une autorité politique étrangère. En attendant une telle évolution bien improbable, il fut décidé de s'intéresser aux modalités pratiques de recrutement des mercenaires et de surveiller tout particulièrement les revues spécialisées et certains sites Internet. Cette vigilance s'imposait d'autant plus que le mode de recrutement s'articule autour de réseaux de connaissance et de solidarité inter-personnels. L'activité de mercenaire n'est pas permanente mais n'en justifie pas moins le recours à des entreprises à géométries variables. Les mercenaires sont des intermittents qui mènent également des activités légales temporaires. Leurs activités entrepreneuriales s'articulent donc dans un enchevêtrement de sociétés-écrans couvrant des activités spécialisées, bien au-delà de la nécessité de confidentialité. Ces entreprises qui procèdent par scissiparité peuvent également servir à contourner les embargos. C'est pourquoi, il est apparu au ministère de la Défense qu'il fallait constituer rapidement en droit un nouveau délit, celui de contournement d'embargo ou tout au moins d'assimiler la violation d'embargo à une circonstance aggravante au plan pénal, pour lutter efficacement contre les mercenaires. En conséquence, la direction des affaires juridiques (DAJ), créée en mars 1999, fut chargée d'établir un répertoire exhaustif de tous les instruments restrictifs en matière d'exportation d'armement adoptés par la République française et de tenir à jour l'enregistrement des modifications intervenues. Les Etats faisant l'objet d'un embargo décidé par l'ONU, l'Union européenne ou l'OSCE sont rappelés chaque année dans l'édition du rapport au parlement sur les exportations d'armement ${ }^{32}$. Si depuis mars 2000, tous les textes et leurs adaptations sont édités (une première), cet acte de transparence ne fut pas jugé suffisant. Une réflexion fut engagée pour examiner la possibilité de créer une nouvelle incrimination pénale et de transposer en droit interne chacun des embargos décidés. L'opposabilité aux personnes morales ou physiques pour caractériser leur intention délictuelle et la mise en action des sanctions pénales appropriées nécessiteraient, toutefois, de publier au Journal officiel, de tenir régulièrement à jour et d'expliciter le champ d'application des mesures décidées, une tâche aussi délicate que minutieuse. Faute de parvenir à un consensus interministériel, ce projet a été abandonné au grand dam du ministre de la Défense qui a souligné que déjà par deux arrêts (30 janvier 1995 18 mai 1998), la Chambre criminelle de la Cour de Cassation a jugé, en se fondant sur les articles 22 et suivants du code des douanes, que faute de mesures de transposition prises en droit interne, les exportations de produits à destination de pays sous embargo, dans le cas présent, l'Irak et la Serbie-Monténégro, ne pouvaient être 
poursuivies sur la base des articles 38, 414 et 428 du code pénal. En attendant de savoir plus précisément si cette décision de justice constitue une véritable jurisprudence susceptible de mettre en cause le principe de l'application directe des règlements communautaires en droit interne et celle des dispositions du code des douanes en matière de sanction (article 38), ces travaux ont révélé d'autres faiblesses du dispositif de contrôle. Alors que de nombreuses résolutions des Nations-Unies et positions communes de l'Union européenne engagent les Etats à prendre les mesures nécessaires pour empêcher de fournir ou une assistance technique concernant la livraison, la fabrication, l'entretien ou l'utilisation d'armements et de matériels connexes et de transporter au moyens de navires battants leur pavillon ou d'aéronefs immatriculés par eux, force est de constater qu'un contrôle satisfaisant n'existe pas en France. Le seul énoncé dans une résolution de ces sanctions ne saurait suffire à dégager l'Etat français de toute responsabilité et constituer un effet dissuasif suffisant, palliant à lui seul l'absence de mécanismes de contrôle ex anteou ex post.Autant de dysfonctionnements auxquels il convient de remédier et qui sont intimement liés à la lutte contre le mercenariat. Les mercenaires ont besoin de moyens de transports, rapides et performants notamment parce que pour conduire leurs opérations ils préfèrent la location à l'acquisition de leurs matériels de guerre compte tenu des coûts d'entretiens trop élevés de ceux-ci. Engager la responsabilité des transporteurs constituerait un moyen de pression supplémentaire et concret. Une fois encore, les mesures envisagées visent d'abord à compliquer la vie de ces acteurs de guerre en leur donnant le sentiment d'être sous surveillance et de pouvoir faire à tout moment l'objet de procédures pénales. Toutes choses étant égales par ailleurs, l'attitude adoptée par les responsables du ministère de la Défense est comparable à celle de ceux qui luttent contre la prostitution. L'interdiction du racolage n'a jamais fait disparaitre les péripatéticiennes, l'interdiction du mercenariat ne permettra pas, à elle seule, de voir disparaître ses pires malandrins. Reste par conséquent à ne pas envisager puis adopter des mesures de contrôles administratifs contre-productives. Pour y parvenir encore faut-il ne pas accorder au mercenariat plus d'importance qu'il n'en mérite. C'est pourquoi au-delà de la loi sur le mercenariat, il apparaissait dans l'entourage du ministre de la Défense la nécessité de mieux prendre en considération la privatisation de la sécurité. Une exigence qui pèse tout autant sur les épaules des responsables du ministère de l'Intérieur (cf. contrôle pacifique des foules) mais pour lesquels l'urgence est toujours apparue moins immédiate (cf. contrôle des exportations de matériels de police et de sécurités3).

Le contrôle des sociétés privées de sécurité est d'autant plus urgent que l'Etat français a de plus en plus de difficultés à répondre aux attentes de sécurité de ses partenaires notamment les plus démunis. Les accords bilatéraux de défense signés au lendemain des indépendances sont d'une valeur politique voire juridique (ex. Comores) incertaine. Les moyens de la coopération militaire et de défense, octroyés par l'administration du ministère des Affaires étrangères, sont en régression tandis que s'effectue un contrôle de plus en plus strict des exportations de matériels de guerre. Non seulement, les approvisionnements au moindre coût s'amenuisent mais les intermédiaires sont sous une surveillance croissante tout comme le transit par l'hexagone des matériels de guerre.

En situation de crise, nombre de partenaires récurrents sont donc convaincus que la France peut être tentée d'adopter une attitude " passive ", devant ce qui lui apparaîtra très certainement comme des imbroglios incompréhensibles. Syndrome rwandais 
oblige, la France ne sera-t-elle pas tentée de rechercher, avant tout, une posture lui permettant de se dégager de toute forme de responsabilité, morale, juridique et politique ? Une conviction largement partagée par ceux qui sont tentés de recourir aux savoir-faire des mercenaires et qui estiment que la France souhaite se désengager des conflits endémiques. Ne sont-ils des guerres sans fin? Même le concept de force africaine de maintien de la paix (RECAMP, ACCRI) attise ce sentiment tout comme la volonté récursive de réduire le nombre de soldats français sous uniformes étrangers. Chez un certain nombre de nos partenaires, on est, en outre, persuadé que la France est à son tour atteinte par le syndrome du body bagou de la théorie américaine du "zéro mort». Dans ce contexte, les Etats qui comptaient autrefois sur le soutien inconditionnel de l'ancienne puissance tutélaire sont tentés de s'équiper au moindre coût et avec des matériels n'appartenant pas au parc français. Il est vrai que les stocks français sont d'autant plus sous surveillance qu'Alain Richard a décidé que toute cession même non onéreuse d'ALPC serait rendue publique par le truchement du rapport annuel au Parlement sur les exportations d'armement. Cette disposition visait à compléter le dispositif administratif visant à un meilleur contrôle des opérations de courtage.

Au-delà de la définition des mercenaires, est, en effet, apparue la nécessité d'une taxinomie plus précise des acteurs non étatiques (entre autres, courtier, coopérant militaire, élément d'une force de sécurité non-gouvernementale...). Dans nombre de conflits mêmes si les mercenaires et les courtiers sont souvent apparus comme deux facettes différentes d'un même métier, il ne s'en est pas moins avéré nécessaire juridiquement de différencier l'un de l'autre. La diffusion incontrôlée des petites armes dans les zones de conflits internes ou frontaliers a incité, de plus, la communauté internationale à accorder une attention particulière à l'action des courtiers en armement qui portent une certaine responsabilité dans cette situation. Ainsi, le courtage a constitué l'une des priorités de la présidence française de l'Union européenne en matière de contrôle des exportations des armes conventionnelles. La France a participé activement aux débats sur ce sujet au sein de l'Union européenne pour définir les principes communs d'une réglementation du courtage. Elle a également été présente dans les instances régionales spécialisées et dans les négociations multilatérales qui ont traité de cette question. Sur le plan intérieur, à l'initiative du Premier ministre L. Jospin, une réflexion administrative a été engagée pour que la France se donne le plus tôt possible tous les moyens de mieux contrôler l'activité des intermédiaires agissant sur son territoire. Ici aussi le droit français s'est dévoilé pour le moins lacunaire. Si, en tant que commerçant, le courtier est soumis à autorisation pour l'exercice de son activité (voir l'article 24 du décret-loi du 18 avril 1939), les opérations de courtage en elles-mêmes, lorsqu'elles ne donnent pas lieu à un mouvement physique d'exportation à partir du territoire national, échappent au contrôle de l'administration. Les travaux interministériels entrepris ont donc visé comme pour les mercenaires à définir les dispositions nécessaires pour pouvoir contrôler et éventuellement, interdire ou sanctionner les opérations de courtage par un renforcement du contrôle a posteriori sur la base de registres spéciaux adaptés aux conditions particulières de l'activité des intermédiaires et des courtiers. A cette fin, le régime d'autorisation préalable des commerçants et fabricants de matériel de guerre a été amendé par le décret 2002-23 du 3 janvier 2002 pour définir la structure de ces registres ${ }^{34}$ et en prévoir la tenue. Le document agréé fait apparaître les différentes parties prenantes et les étapes successives des opérations concernées, facilitant ainsi la vérification par le Contrôle 
général des armées (CGA) et les officiers de police judiciaire qui lui sont affectés, les transactions réalisées et la poursuite des infractions éventuelles. Ces documents permettent ainsi de savoir si des mercenaires s'approvisionnent en armes notamment de petits calibres. Comme dans le texte de loi visant les mercenaires, l'ensemble du régime de contrôle de l'intermédiation a été conçu pour s'appliquer aux personnes résidentes ou établies en France.

Le cadre juridique du contrôle a posteriori a été rapidement défini car il ne portait que sur une réforme réglementaire. L'instauration d'un contrôle a priori était une réforme de plus grande ampleur qui nécessitait notamment pour l'assortir de sanctions pénales adaptées de passer par la voie parlementaire. Une deuxième étape de la manœuvre qu'un calendrier parlementaire trop chargé ne permit pas de mener à bien, la priorité gouvernementale étant alors accordée au dépôt du texte visant les mercenaires. Il n'en reste pas moins que l'institution d'un contrôle a priori offrirait à l'administration la possibilité d'interdire une opération de courtage ou de soumettre sa réalisation à des conditions particulières. Un projet qui semble depuis deux ans en suspens. Ce système procurait pourtant l'avantage d'esquisser les premières mesures d'encadrement des sociétés privées de sécurité en se traduisant par la délivrance d'autorisations au coup par coup ou de licences plus générales pour une catégorie d'opérations. Une procédure interministérielle d'examen préalable aurait permis, de jure, de recueillir l'avis des administrations compétentes sur chaque dossier et de l'élargir peu à peu à toutes les autres activités commerciales des sociétés de sécurité. Tout aussi important, le régime envisagé devait donner à l'administration les moyens de contrôler cette activité jusqu'à présent mal cernée, tout en évitant d'entraver le commerce licite ou de gêner l'action des intermédiaires respectant les dispositions légales. En d'autres termes, ces dispositions législatives associant un mécanisme de police administrative (comme par exemple l'obligation de déclaration) et des sanctions pénales importantes sont indispensables pour une lutte totale contre les mercenaires.

Dans ce contexte, plus que jamais, il faut distinguer très nettement l'assistance militaire durable, publique et privée, et l'activité des mercenaires. Ceci passe immanquablement par une réflexion plus approfondie sur les compagnies militaires privées, même si leurs activités rencontrent tout particulièrement de la méfiance en France ${ }^{35}$. Dans l'hexagone, elle est à peine esquissée (voir le rapport du général Heinrich au ministre de la Défense, 2002) pourtant la continuité entre ces nouvelles compagnies militaires privées et l'action des mercenaires n'est même pas niée par leurs zélateurs $^{36}$ et nous ne sommes plus au XVIIIème siècle quand la Compagnie anglaise des Indes orientales conduisait encore ses guerres selon ce modèle. Définir le cadre juridique de leurs activités est d'autant plus urgent que l'on voit des mercenaires s'ouvrir à de nouveaux métiers d'influence tels la cyberguerre ${ }^{37}$, l'interprétation d'imagerie satellitaire achetée sur les marchés civil ${ }^{38}$ voire la lutte anti-terroriste. La privatisation du maintien de la paix pourrait leur assurer également de belles perspectives. N'offrent-ils pas l'avantage d'assurer un déploiement rapide, voire "peu» coûteux ${ }^{39}$ ? La réglementation de ces activités en expansion se justifie également du point de vue juridique. La législation relative aux sociétés fournissant des services dans le domaine de la sécurité se limite à régir pour l'heure les activités de recherche privée et les activités privées de surveillance, de gardiennage et de transports de fonds ${ }^{40}$. De plus, une lecture un peu «audacieuse » de l'article 436-2 du Code pénal pourrait conduire à l'interdiction des sociétés militaires privées ${ }^{41}$. Une situation encore bien éloignée des espoirs de E. Kant qui espérait une percée 
prometteuse de paix perpétuelle avec l'avènement d'une constitution républicaine où il n'y aurait plus ni mercenaires, ni armée de métier. Reste à savoir si une Commission d'exportation des services de sécurité sur le modèle de la Commission interministérielle pour l'étude des exportations de matériel de guerre (CIEEMG) constituerait la réponse la plus appropriée à l'«entrepreneuralisation» des mercenaires! Une chose est sûre, la réglementation européenne ne sera pas, ici, d'un grand secours. Elle n'existe pas et l'espoir de voir notre législation inspirer celles de nos partenaires européens ${ }^{42}$ est un vœu pieux pour l'heure puisque à la différence du courtage qui est évoqué dans de nombreux forums internationaux ${ }^{43}$, le mercenariat et ses nouvelles formes entrepreneuriales ne sont pas débattus dans les instances européennes compétentes.

\section{NOTES}

1. Christian Leverchy est conseiller pour les Affaires internationales du ministre de la Défense (1997-2002). Les propos et appréciations exprimés ici n'engagent que leur seul auteur.

2. . On dit qu' «Un homme est mercenaire, qu'il a l'ame mercenaire, pour dire qu'Il se laisse aisément corrompre à l'interest, qu'on luy fait faire tout ce qu'on veut pour de l'argent ».

3. . Intervention du député Frédéric Dutoit (PCF) le 3 avril 2003 à l'Assemblée nationale. (http://www.bdr13.pcf.fr/documents/document.phtml ?iddoc=1189).

4. . Loi $n^{\circ}$ 2003-340 du 14 avril 2003 relative à la répression de l'activité de mercenariat, Journal Officiel, n89 du 15 avril 2003, p. 6636.

5. . http://www.senat.fr/seances/s200302/s.200030206/s20030206004.html, p. 2.

6. . Le Matin des Comores, 15 mars 2003.

7. . Le travail interministériel devait être terminé pour le début de l'année 2001. http:// www.defense.gouv.fr/actualités/dossier/d95/raparm.pdf, p. 11.

8. . Au 15 janvier 2004, une seule procédure semble avoir été engagée.

9. . http://www.assemblee-nat.fr/12/cri/2002-2003/20030185.asp, p. 3.

10. . Jean Marguin, «La privatisation des forces armées : une évolution inéluctable ?", L'Armement, n69/2000 ; Jean-Philippe Daniel, La politique militaire de la France au Sud du Sahara, du discours de la Baule à l'opération Hadès : du désengagement à la privatisation, Mémoire de DEA, Université de Paris 1, 2000.

11. . G2S, « La France en Afrique : Deux opérations extérieures riches d'enseignements », Lettre d'information, novembre 2003, p.5.

12. . Cette activité est tout aussi « légitimisante » que militaire puisqu'elle met les mercenaires au contact avec des organisations non gouvernementales à vocation humanitaire et en fait de nouveaux acteurs de bien.

13. Jean de Tonquedec, Marchands d'armes, Flammarion, Paris, 2003, p. 132.

14. . François Dominguez, Barbara Vignaux, «La nébuleuse des mercenaires français », Le Monde Diplomatique, août 2003. 
15. . Daniel Burton-Rose, Wayne Madsen, « Corporate Soldiers : The US Government Privatizes the Use of Force », Multinational Monitor, vol. 20, 3 mars 1999.

16. . Pierre Conesa, « Modernes mercenaires de la sécurité », Le Monde Diplomatique, avril 2003.

17. . http://www.senat.fr/seances/s200302/s20030206/s20030206004.html

18. . http://www.archives.premier-ministre.gouv.fr/jospin_version3/fr/ie4/contenu/ 5137.htm, p.11.

19. . http://www.assemblee-nat.fr/12/propositions/pion0499.asp

20. . Loi n²001-79 du 30 janvier 2001, Journal Officiel, 30 juin 2001.

21. . Campagne Mercenaires : Halte là ! (http://www.fraternet.org/survienord/ halte.html).

22. . 9ème rapport du Foreign Affairs Committee de la Chambre des Communes, Private Military Companies : Option for Regulation, Foreign and Commonwealth Office, 1er août 2002 (http://www.fco.gov.uk).

23. . Cette structure fit l'objet d'une commission d'enquête présidée par Guy Hermier (PCF) (http://www.assemblee-nationale.fr/dossiers/dps.asp).

24. . Rapport au Parlement sur les exportations de la France - Résultats 1999, (http:// www.defense.gouv.fr/actualités/dossier/d95/raparm.pdf, p.10).

25. . Rapport de M. Marc Jourlaud sur le projet de loi, adopté par le Sénat, relatif à la répression de l'activité de mercenaire (http://www.assemblée-nat.fr/12/rapports/ r0671.asp, p. 8).

26. . Eric David, « Les mercenaires en droit international », Revue belge de droit international public, vol. 13/1977 ; Philippe Chapleau, François Misser : Mercenaires S.A., Desclée de Brouwer, Paris, 1998, 220 p.

27. . L'assimilation de la Légion étrangère à un corps de mercenaires n'est pas rare. Les citoyens polonais qui ont fait le choix de servir ainsi la France, sont ainsi susceptibles de poursuites judiciaires dans leur pays d'origine. Dans les milieux universitaires étrangers, cette appréciation est tout aussi fréquente. On la trouve par exemple sous la plume du professeur de sciences politiques de l'université berlinoise de Humboldt, Herfried Münkler dans son dernier ouvrage Les guerres nouvelles (Alvik Editions, Paris, 2003, p. 40).

28. . http://senat.fr/seances/s200302/s20030206/s20030206004.html, p. 9.

29. . http://www.assemblee-nat.fr/12/rapports/r0671.asp, p. 17.

30. . http://senat.fr/rap/102-142/102-142_mono.html, p. 12.

31. . Christian Lechervy, « Le ministère de la Défense et l'utilisation des mercenaires » in Survie : Le boom du mercenariat. Défi ou fatalité, Damoclès, Lyon, 2001, p. 56.

32. . Pour la dernière édition de rapport : Rapport au Parlement sur les exportations d'armement de la France en 2001, Ministère de la Défense, Paris, juin 2003, pp. 71-82.

33. . Une liste de matériels de police et de sécurité (MPS) qui devraient faire l'objet d'un contrôle à l'exportation a été élaborée par les Etats membres de l'Union européenne dans le cadre du COARM et transmise en décembre 2000 à la Commission à qui il revient de proposer au Conseil un projet de règlement communautaire.

34. . Le modèle de registre spécial des opérations d'intermédiation découle des obligations fixées par l'arrêté du 20 mars 2002. NOR : DEFCo2011317A.

35. . « De la guerre au nouveau business mercenaire ", Critique internationale, $\mathrm{n}^{\circ} 1$, Paris, automne 1998, pp. 179-194.

36. . « Mercenaires : Des chiens de guerre aux sociétés étatiques », Raids, n¹96, septembre 2002 . 
37. Jacques Château, La fin de l'ordre militaire et le retour des mercenaires, Institut d'Etudes Politiques, Aix-en-Provence, 2001, p. 117.

38. . Major Thomas J. Milton, The New Mercenaries - Corporate Armies for Hire, Foreign Area Officer, Sringfield, 1997 (http://www.faoa.org).

39. . Une étude du South African Institute of International Affairs évaluait à un milliard d'euro le coût du retour à la paix en Afrique par l'entremise de compagnies privées de sécurité. Cf. Peter Fabricius, « Private Security Firms Can End Africa's War Cheaply », Saturday Star, (Johannesbourg), 23 septembre 2000.

40. . Actuellement réglementées par la loi n83-629 du 12 juillet 1983, celle-ci fixe le régime administratif de ces activités, les conditions d'exercice de la profession et du contrôle administratif des entreprises.

41. . Aymeric Philipon, Le mercenaire et le droit, p. 8. (texte non référencé). Voir également « Les activités mercenaires et le droit ", $n^{\circ} 24$, Recherches et Documents, voir site de la Fondation pour la Recherche Stratégique, FRS (http://www.frstrategie.org). 42. . Le sénateur Robert Bret (membre du groupe Communiste, Républicain et Citoyen) lors du débat parlementaire du 6 février se montrera plus explicite encore en visant tout particulièrement la Grande Bretagne, tout comme le député Paul Quilès (PS) et les nouveaux partenaires de l'Union (http://www.robertbret.org/article.php3 ?

id_article=101). La ministre elle-même lors de la séance du 3 avril 2003 mit en cause de « véritables entreprises de guerre, souvent d'origine anglo-saxonne » tandis que le député Axel Poniatowski (UMP) exprima le souhait que ces dispositions encouragent nos partenaires « d'outre-atlantique » à prendre des dispositions allant dans le même sens.

43. . Au niveau international la question du contrôle des opérations d'intermédiation a été abordée au sein de l'ONU (ex. protocole sur les armes à feu additionnel à la convention sur la criminalité transnationale organisée et groupe d'experts et au groupe d'experts préparant la conférence de 2001 sur le commerce illicite des armes légères et de petits calibres), à l'OSCE (voir le document sur les ALPC du 23 novembre 2000, dans le cadre de l'arrangement de Wassenaar sur le contrôle des exportations d'armes conventionnelles et de biens et technologies à double usage et dans le cadre du groupe COARM de l'Union européenne où furent adoptées, en 2001, des lignes directrices pour le contrôle du courtage.

\section{INDEX}

Index géographique : France

Mots-clés : droit, privatisation de la sécurité 